\title{
BOUNDARY BEHAVIOR OF LARGE SOLUTIONS TO QUASILINEAR ELLIPTIC PROBLEMS WITH A NONLINEAR GRADIENT TERM
}

\author{
ChUnlian LiU AND ZuOdONG YANG
}

Abstract. In this paper,we study the boundary behavior of solutions to boundary blow-up elliptic problems

$$
\left\{\begin{array}{l}
\operatorname{div}\left(|\nabla u|^{m-2} \nabla u\right) \pm|\nabla u(x)|^{q(m-1)}=b(x) f(u), \quad x \in \Omega, \\
u>0, \quad x \in \Omega \\
\left.u\right|_{\partial \Omega}=+\infty
\end{array}\right.
$$

where $\Omega$ is a bounded domain with smooth boundary in $\mathbb{R}^{N}, m>1, q>0, b \in C^{\alpha}(\bar{\Omega})$, which is positive in $\Omega$ and may be vanishing on the boundary and rapidly varying near the boundary, and $f$ is rapidly varying or normalized regularly varying at infinity.

Mathematics subject classification (2010): 35J25, 35B50, 35J40.

Keywords and phrases: Large solutions, quasilinear elliptic equation, asymptotic behavior, boundary blow-up, nonlinear gradients.

\section{REFERENCES}

[1] C. Bandle, E. Giarrosso, Boundary blow-up for semilinear elliptic equations with nonlinear gradient terms, Adv. Differential Equations, 1 (1996), 133-150.

[2] C. Bandle, M. Marcus, Large solutions of semilinear elliptic equations: existence, uniqueness and Asymptotic behavior, J. Anal. Math., 58 (1992), 9-24.

[3] F. Cîrstea, V.D. Rădulescu, Existence and Uniqueness of blow-up boundary solution of logistic equations, Commun. Contemp. Math., 4 (2002), 559-586.

[4] F. Cîrstea, V.D. Rădulescu, Uniqueness of the blow-up boundary solution of logistic equations with absorbtion, C. R. Acad. Sci. Paris Ser.I., 335 (2002), 447-452.

[5] M. Ghergu, C. Niculescu and V. Rădulescu, Explosive solutions of elliptic equations with absorption and non-linear gradient term, Proceedings of the Indian Academy of Sciences. Mathematical Sciences, 112, (3) (2002), 441-451.

[6] M. Ghergu, V. Rădulescu, Nonradial blow-up solutions of sublinear elliptic equations with gradient term, Communications on Pure and Applied Analysis, 3, (3) (2004), 465-474.

[7] A.V. Lair, A.W. Shaker, Classical and weak solutions of a singular semilinear elliptic problem, J. Math. Anal. Appl., 211 (1997), 371-385.

[8] A.V. Lair, A necessary and sufficient condition for existence of large solutions to semilinear elliptic equations, J. Math. Anal. Appl., 240 (1999), 205-218.

[9] A.V. Lair, A.W. Wood, Large solutions of semilinear elliptic equations with nonlinear gradient terms, International Journal of Mathematics and Mathematical Sciences, 22, (4) (1999), 869-883.

[10] C. Liu, Z. Yang, Blow-up rates of large solutions for quasilinear elliptic equations, British Journal of Mathematics and Computer Science 3, (4) (2013), 605-616.

[11] C. Liu, Z. Yang, Existence of large solutions for quasilinear elliptic problems with a gradient term, Applied Mathematics and Computation, 192 (2007), 533-545.

[12] C. Liu, Z. Yang, Boundary blow-up quasilinear elliptic problems of the Bieberbach type with nonlinear gradient terms, Nonlinear Anal., 69 (2008), 4380-4391.

[13] C. Liu, Z. Yang, Boundary blow-up quasilinear elliptic problems with nonlinear gradient terms, Complex Variables and Elliptic Equations, 57, (6) (2012), 687-704. 
[14] Q. Lu, Z. Yang, E.H. Twizell, Existence of entire explosive positive solutions of quasilinear elliptic equations, Applied Mathematics and Computation, 148 (2004), 359-372.

[15] V. Maric, Regular variation and differential equations,in: Lecture Notes in Mathematics, Vol.1726, Springer, 2000.

[16] R. Osserman, On the inequality $\Delta u \geqslant f(u)$, Pacific J. Math., 7 (1957), 1641-1647.

[17] S.I. Resnick, Extreme Values, Regular Variation, and Point Processes, Springer-Verlag, New York, 1987.

[18] R. Seneta, Regular varying functions, in:Lecture Notes in Mathematics, Vol.508, Springer, 1976.

[19] F. Toumi, Existence of blow-up solutions for nonlinear problems with a gradient term, International Journal of Mathematics and Mathematical Sciences 2006 (2006), 1-11.

[20] Z. Yang, Existence of explosive positive solutions of quasilinear elliptic equations, Applied Mathematics and Computation, 177 (2006), 581-588.

[21] Z. Zhang, Boundary behavior of large solutions to semilinear elliptic equations with nonlinear gradient terms, Nonlinear Anal., 73 (2010), 3348-3363. 